\title{
Weak Separation, Pure Domains and Cluster Distance
}

\author{
Miriam Farber $\|$ and Pavel Galashin $\|^{1}$ \\ ${ }^{1}$ Department of Mathematics, Massachusetts Institute of Technology, Cambridge MA, USA.
}

\begin{abstract}
Following the proof of the purity conjecture for weakly separated sets, recent years have revealed a variety of wider classes of pure domains in different settings. In this paper we show the purity for domains consisting of sets that are weakly separated from a pair of "generic" sets $I$ and $J$. Our proof also gives a simple formula for the rank of these domains in terms of $I$ and $J$. This is a new instance of the purity phenomenon which essentially differs from all previously known pure domains. We apply our result to calculate the cluster distance and to give lower bounds on the mutation distance between cluster variables in the cluster algebra structure on the coordinate ring of the Grassmannian. Using a linear projection that relates weak separation to the octahedron recurrence, we also find the exact mutation distances and cluster distances for a family of cluster variables.

Résumé. Suite à la preuve de la conjecture de pureté sur les ensembles faiblement séparés, des familles variées de domaines pures sont apparues récemment dans différents contextes. Dans cet article, nous prouvons la pureté de domaines formés par les ensembles qui sont faiblement séparés d'une paire d'ensembles "génériques" $I$ et $J$. Notre preuve donne aussi une formule simple pour le rank de ces domaines en termes de $I$ et $J$. Il s'agit d'une nouvelle instance du phénomène de pureté qui diffère essentiellement de tous les domaines pures connus précédemment. Nous appliquons notre résultat pour calculer la distance d'amas et pour donner des bornes inférieures sur la distance de mutation entre les variables d'amas dans la structure d'algèbre amassée sur l'anneau de coordonnées de la Grassmannienne. En utilisant une projection linéaire qui relie la séparation faible à la récurrence de l'octaèdre, nous trouvons aussi les distances de mutation et les distances d'amas exactes pour une famille de variables d'amas.
\end{abstract}

Keywords. weak separation, purity conjecture, cluster distance, mutation sequences

\section{Introduction}

In 1998, Leclerc and Zelevinsky introduced the notion of weakly separated collections while studying quasicommuting families of quantum minors (see Leclerc and Zelevinsky (1998)). They raised the "purity conjecture", which states that all maximal by inclusion collections of pairwise weakly separated subsets of $[n]:=\{1,2 \ldots, n\}$ have the same cardinality. This conjecture was proven independently by both $\mathrm{Oh}$ et al. (2015) and Danilov et al. (2010). Since then, it motivated the search for a wider classes of pure domains. Such domains have been found (Danilov et al. (2014)), using a novel geometric-combinatorial

\footnotetext{
${ }^{\dagger}$ Email: mfarberemit.edu. This author is supported by the National Science Foundation Graduate Research Fellowship under Grant No. 1122374.

‡Email: galashin@mit.edu

1365-8050 @ 2016 Discrete Mathematics and Theoretical Computer Science (DMTCS), Nancy, France
} 
model called combined tilings. Furthermore, the work of Oh et al.(2015) showed that all maximal weakly separated collections can be obtained from each other by a sequence of mutations. In particular, this result implies that sets of Plücker coordinates labeled by maximal weakly separated collections form clusters in the cluster algebra structure on the coordinate ring of the Grassmannian. All the pure domains that were studied so far have their maximal weakly separated collections lying inside or outside a simple closed curve (we will recall the definition of this later). In this paper, we discover a new type of pure domains associated with pairs of subsets of $[n]$. For these domains the structure of maximal weakly separated collections is of a different nature than in all of the above cases. Such domains arise naturally while studying a notion of cluster distance between two cluster variables, and specifically variables that are Plücker coordinates in the cluster algebra structure on the coordinate ring of the Grassmannian. The purity of these domains allows us to compute this distance for any "generic" pair of cluster variables. We further reformulate this distance in the context of general cluster algebras.

Let us first motivate the need for a notion of distance in a cluster algebra, concentrating on the example of the Grassmannian. A pair of Plücker coordinates can appear together in the same cluster iff they are labeled by weakly separated subsets of $[n]$. What about Plücker coordinates that do not appear together in the same cluster? We would like to estimate how close they are to being weakly separated. In a more general sense, it would be beneficial to have a notion that would measure how close are two cluster variables from appearing in the same cluster. When they do appear in the same cluster we say that the distance between them is zero. Section 2 develops this notion (defined in equation (1p) and introduces the domains mentioned above. Section 3 introduces our main result. Section 4 provides the necessary background on domains inside and outside simple closed curves, used in the sketch of the proof of our main result in Section 5. Finally, Section 6 gives a formula for the mutation distance (introduced in Farber and Postnikov (2015)) for a family of pairs of cluster variables and relates the corresponding optimal sequence of mutations with that of the octahedron recurrence.

\section{Preliminaries}

For $0 \leq k \leq n$, we denote by $\left(\begin{array}{c}{[n]} \\ k\end{array}\right)$ the collection $\left.{ }^{(\text {i) }}\right]$ of $k$-element subsets of $[n]$. For two subsets $I, J \subset[n]$ we write $I<J$ if $\max (I)<\min (J)$. We say that $I$ surrounds $J$ if $|I| \leq|J|$ and $I \backslash J$ can be partitioned as $I_{1} \sqcup I_{2}$ such that $I_{1}<J \backslash I<I_{2}$. We denote by $I \triangle J$ the symmetric difference $(I \backslash J) \cup(J \backslash I)$.

Definition 2.1 (Leclerc and Zelevinsky (1998)) Two sets $I$ and $J$ are said to be weakly separated if either I surrounds $J$ or J surrounds I (or both).

This definition has a particularly simple meaning when $I$ and $J$ have the same size. Consider a convex $n$-gon with vertices labeled by numbers 1 through $n$. Then it is easy to see that two subsets $I$ and $J$ of the same size are weakly separated iff the convex hull of the vertices from the set $I \backslash J$ does not intersect the convex hull of the vertices from the set $J \backslash I$. A collection $\mathcal{C} \subset 2^{[n]}$ is called weakly separated if any two of its elements are weakly separated.

Definition 2.2 (Danilov et al. (2014)) A collection $\mathcal{A} \subset 2^{[n]}$ is called a pure domain if all maximal (by inclusion) weakly separated collections of sets from $\mathcal{A}$ have the same size. In this case, the size of all such collections is called the rank of $\mathcal{A}$ and denoted $\operatorname{rk} \mathcal{A}$.

(i) throughout the paper, we reserve the word "set" for subsets of $[n]$ while we use the word "collection" for subsets of $2^{[n]}$. 
The following two surprising results go under the name "purity phenomenon" and were conjectured in Leclerc and Zelevinsky (1998) and Scott (2005), respectively. Both of them were proven independently in Oh et al. (2015) and in Danilov et al. (2010):

Theorem 2.3 The collection $2^{[n]}$ is a pure domain of rank $\left(\begin{array}{l}n \\ 2\end{array}\right)+n+1$.

Theorem 2.4 The collection $\left(\begin{array}{c}{[n]} \\ k\end{array}\right)$ is a pure domain of rank $k(n-k)+1$.

The latter result has a stronger version that relates pairs of maximal (by inclusion) weakly separated collections by an operation that is called a mutation.

Proposition 2.5 (Leclerc and Zelevinsky (1998), Scott (2005)) Let $S \in\left(\begin{array}{c}{[n]} \\ k-2\end{array}\right)$ and let $a, b, c, d$ be cyclically ordered elements of $[n] \backslash S$. Suppose a maximal weakly separated collection $\mathcal{C}$ contains $S a b, S b c, S c d, S d a$ and Sac. Then $\mathcal{C}^{\prime}=(\mathcal{C} \backslash\{S a c\}) \cup\{S b d\}$ is also a maximal weakly separated collection.

Here by $S a b$ we mean $S \cup\{a, b\}$. We say that $\mathcal{C}^{\prime}$ and $\mathcal{C}$ as above are connected by a mutation (ii)

Theorem 2.6 (Oh et al. (2015)) $\left(\begin{array}{c}{[n]} \\ k\end{array}\right)$ is a pure domain of rank $k(n-k)+1$. Moreover, any two maximal weakly separated collections in $\left(\begin{array}{c}{[n]} \\ k\end{array}\right)$ are connected by a sequence of mutations.

The list of collections of subsets of $[n]$ known to be pure domains is not restricted to just $2^{[n]}$ and $\left(\begin{array}{c}{[n]} \\ k\end{array}\right)$. In Oh et al. (2015) both Theorem 2.3 and Theorem 2.4 above can be seen as special cases of the purity phenomenon for the collections inside a positroid, while in Danilov et al. (2010) these collections are inside and outside of a generalized cyclic pattern. Note that in both Oh et al. (2015) and Danilov et al. (2010), the collections are required to lie, in a sense, inside and outside a specific simple closed curve (we will define that more rigorously later). In this paper we present a new instance of the purity phenomenon which does not fit into this context. The domains that we discuss arise naturally when dealing with distances between cluster variables in the cluster algebra on the coordinate ring of the Grassmannian.

Definition 2.7 For $n \geq k \geq 0$, the Grassmannian $G r(k, n)$ (over $\mathbb{R})$ is the space of $k$-dimensional linear subspaces in $\mathbb{R}^{n}$. It can be identified with the space of real $k \times n$ matrices of rank $k$ modulo row operations. The $k \times k$ minors of $k \times n$-matrices form projective coordinates on the Grassmannian, called the Plücker coordinates, that are denoted by $\Delta_{I}$, where $I \in\left(\begin{array}{c}{[n]} \\ k\end{array}\right)$.

Theorem 2.6 implies the following (Oh et al. (2015)):

Theorem 2.8 For $\mathcal{C} \subset\left(\begin{array}{c}{[n]} \\ k\end{array}\right), \mathcal{C}$ is a maximal weakly separated collection iff the set of Plücker coordinates $\left\{\Delta_{I}\right\}_{I \in \mathcal{C}}$ is a cluster in the cluster algebra structure on the coordinate ring of $G r(k, n)$.

This theorem associates maximal weakly separated collections with clusters, and $k$-tuples in $\left(\begin{array}{c}{[n]} \\ k\end{array}\right)$ with cluster variables, which are the Plücker coordinates. Two cluster variables can appear in the same cluster iff they are weakly separated. This trait leads to the following natural question: Given any pair of cluster variables - how far are they from appearing in the same cluster? More formally, let $I, J \in\left(\begin{array}{c}{[n]} \\ k\end{array}\right)$. Define:

$$
d(I, J)=k(n-k)+1-\max \left\{\left|\mathcal{C}_{1} \cap \mathcal{C}_{2}\right|: \mathcal{C}_{1}, \mathcal{C}_{2} \subset\left(\begin{array}{c}
{[n]} \\
k
\end{array}\right) \text { and } I \in \mathcal{C}_{1}, J \in \mathcal{C}_{2}\right\}
$$

\footnotetext{
(ii) Such a mutation is usually called a square move. There is a more general notion of a cluster mutation, but it is beyond the scope of this paper.
} 
such that both $\mathcal{C}_{1}$ and $\mathcal{C}_{2}$ on the RHS are weakly separated collections. Theorem 2.4 implies that $d(I, J)=$ 0 iff $I$ and $J$ are weakly separated, in which case we can take $\mathcal{C}_{1}=\mathcal{C}_{2}$ to be any maximal weakly separated collection containing $I$ and $J$. Thus, $d(\cdot, \cdot)$ measures how close a pair of $k$-tuples is to being weakly separated. This notion can be extended to any cluster algebra, by replacing $k(n-k)+1$ with the rank of the algebra and $\mathcal{C}_{1}$ and $\mathcal{C}_{2}$ to be clusters. This defines a distance between cluster variables. Another (and different) notion of distance between clusters was studied in Oh and Speyer (2014).

For a fixed $n$, we say that $I \subset[n]$ is an interval if $I$ is of the form $[a, b]=\{a, a+1, \ldots, b-1, b\}$. If $b<a$ then we consider the elements in $[a, b]$ modulo $n$. For example, if $n=6$ then $[2,4]=\{2,3,4\}$ and $[5,2]=\{5,6,1,2\}$. Note that $d(\cdot, \cdot)$ does not satisfy the triangle inequality since if $I$ is an interval then $d(I, J)=0$ for any $J$. In addition, both $\mathcal{C}_{1}$ and $\mathcal{C}_{2}$ always contain the following $n$ boundary sets:

$$
\mathcal{B}_{k, n}=\{\{1,2, \ldots, k\},\{2,3, \ldots, k+1\}, \ldots,\{1,2, \ldots, k-1, n\}\}
$$

and thus $0 \leq d(I, J) \leq k(n-k)+1-n=(k-1)(n-k-1)$ for any pair $I, J \in\left(\begin{array}{c}{[n]} \\ k\end{array}\right)$.

Definition 2.9 For any pair of $k$-tuples $I, J \in\left(\begin{array}{c}{[n]} \\ k\end{array}\right)$ we define the domain $\mathcal{A}_{I, J} \subset\left(\begin{array}{c}{[n]} \\ k\end{array}\right)$ to be the collection of all $k$-tuples that are weakly separated from both I and $J$.

We can now define $d(\cdot, \cdot)$ equivalently as follows:

$$
d(I, J)=k(n-k)+1-\max \left\{|\mathcal{C}|: \mathcal{C} \subset \mathcal{A}_{I, J} \text { is weakly separated }\right\} .
$$

The optimal collection $\mathcal{C}$ on the RHS of (2) would be just $\mathcal{C}_{1} \cap \mathcal{C}_{2}$ for the optimal pair $\mathcal{C}_{1}$ and $\mathcal{C}_{2}$ in (1).

Example 2.10 Let $I=\{1,2,4\}, J=\{3,5,6\} \in\left(\begin{array}{c}{[6]} \\ 3\end{array}\right)$. Since $I$ and $J$ are not weakly separated, $\max \left\{|\mathcal{C}| \mid \mathcal{C} \subset \mathcal{A}_{I, J}\right.$ is weakly separated $\}<k(n-k)+1=10$. This maximum actually equals 8 and is achieved at $\mathcal{C}=\mathcal{B}_{3,6} \cup\{\{1,2,5\},\{1,3,4\}\}$. Therefore, $d(I, J)=k(n-k)+1-8=2$.

Let us consider another pair $\widehat{I}=\{1,3,5\}, \widehat{J}=\{2,4,6\} \in\left(\begin{array}{c}{[6]} \\ 3\end{array}\right)$. This time, the maximal size of a weakly separated collection in $\mathcal{A}_{\widehat{I}, \widehat{J}}$ is 6 and the only such collection is just $\mathcal{B}_{3,6}$. Hence, $d(\widehat{I}, \widehat{J})=$ $(k-1)(n-k-1)=4$. Note that this value is the largest one that $d(\cdot, \cdot)$ can take for $n=6$ and $k=3$. This example can be generalized to any $k$ with $n=2 k:$ for $I:=\{1,3, \ldots, 2 k-1\}$, we will see in Lemma 5.6 that $\mathcal{A}_{I, \bar{I}}=\mathcal{B}_{k, 2 k}$ and thus $d(I, \bar{I})=(k-1)(n-k-1)$.

Thus finding the maximal size of a weakly separated collection in $\mathcal{A}_{I, J}$ enables us to calculate $d(I, J)$. In this paper, we go further and show that for any "generic" pair $I$ and $J, \mathcal{A}_{I, J}$ is a pure domain, and we find its rank. This not only gives us the value of $d(I, J)$ for such pairs, but also introduces a new class of pure domains with a different structure from the previously known classes.

\section{Main result}

In this section we introduce our main result, which deals with the purity of $\mathcal{A}_{I, J}$ for balanced pairs $I, J$. We first discuss the case in which $I$ and $J$ form a complementary pair of sets (so $I \in\left(\begin{array}{c}{[2 k]} \\ k\end{array}\right)$ and $J=[2 k] \backslash I$ ) and then proceed to the general case. 


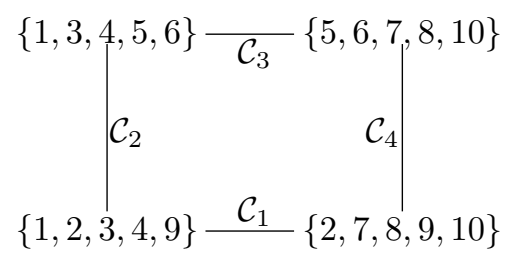

Fig. 1:

Elements of $\mathcal{A}_{I, \bar{I}} \backslash \mathcal{B}_{5,10}$. Edges correspond to weakly separated pairs.

Definition 3.1 Fix any integer $k$ and let $I \in\left(\begin{array}{c}{[2 k]} \\ k\end{array}\right)$ be a set. Then $I$ and its complement $\bar{I}$ partition the circle (with numbers $1,2, \ldots, 2 k$ ) into an even number of intervals $I=P_{1} \cup P_{3} \cup \ldots \cup P_{2 r-1}$, $\bar{I}=P_{2} \cup P_{4} \cup \ldots \cup P_{2 r}$ for some $r \geq 1$, where $P_{i}$ are intervals for any $1 \leq i \leq r$ and $P_{1}<P_{2}<$ $P_{3}<\cdots<P_{2 r-1}<P_{2 r}$ (these inequalities are "modulo $2 k$ "). We say that $\left\{P_{i}\right\}_{i=1}^{2 r}$ are the intervals associated with $I$. We also let $p_{i}:=\left|P_{i}\right|$ be their cardinalities and say that $\left(p_{1}, p_{2}, \ldots, p_{2 r}\right)$ is the partition of the circle associated with $I$. We say that $I$ is balanced if for any $i \neq j \in[2 r]$ we have $p_{i}+p_{j}<k$.

For an even number $t$, we denote by $\mathcal{P}_{t}$ the collection of all sets $I$ for which $2 r=t(2 r$ is taken from the definition above). $I$ and $\bar{I}$ are weakly separated iff $I \in \mathcal{P}_{2}$. In addition, note that if $I \in \mathcal{P}_{4}$ then it is not balanced, since $p_{1}+p_{3}=p_{2}+p_{4}=k$. We study the structure of $\mathcal{A}_{I, \bar{I}}$ and $d(I, \bar{I})$ for $I \in \mathcal{P}_{4}$ in the last section, and relate them to the octahedron recurrence.

Example 3.2 Let $I=\{1,2,4,6,8\}, \bar{I}=\{3,5,7,9,10\} \in\left(\begin{array}{c}{[10]} \\ 5\end{array}\right)$. Then $I$ and $\bar{I}$ partition the circle into the following intervals associated with $I:\{1,2\},\{3\},\{4\},\{5\},\{6\},\{7\},\{8\},\{9,10\}$. Therefore, I $\in \mathcal{P}_{8}$ and $(2,1,1,1,1,1,1,2)$ is the partition of the circle associated with $I$. Since both $2+1$ and $2+2$ are smaller than $5, I$ is balanced.

Theorem 3.3 Let $I \in\left(\begin{array}{c}{[2 k]} \\ k\end{array}\right)$ be balanced and let $\left(p_{1}, p_{2}, \ldots, p_{2 r}\right)$ be the partition of the circle associated with I. Then $\mathcal{A}_{I, \bar{I}}$ is a pure domain of rank

$$
2 k+\sum_{i=1}^{2 r}\left(\begin{array}{c}
p_{i} \\
2
\end{array}\right) .
$$

Remark 3.4 This theorem fails for some non-balanced sets. But note that if the set I is chosen uniformly at random then it is clear that I will be balanced with probability close to 1 for large values of $k$, so the "balancedness" property can be thought of as the analogue of being a "generic" set.

Example 3.5 Let $I, \bar{I}$ be as in Example 3.2. Then

$$
\mathcal{A}_{I, \bar{I}}=\mathcal{B}_{5,10} \cup\{\{1,2,3,4,9\},\{1,3,4,5,6\},\{2,7,8,9,10\},\{5,6,7,8,10\}\} .
$$

Consider a graph with vertex set $\mathcal{A}_{I, \bar{I}}$ and an edge between $S$ and $T$ iff $S$ and $T$ are weakly separated. Then the vertices labeled by the elements of $\mathcal{B}_{5,10}$ are connected to each other and to all other vertices and the remaining four vertices form the square in Figure 1 The are no triangles in Figure 1, so there are 4 maximal weakly separated collections in $\mathcal{A}_{I, \bar{I}}$ : 


$$
\begin{gathered}
\mathcal{C}_{1}=\mathcal{B}_{5,10} \cup\{\{1,2,3,4,9\},\{2,7,8,9,10\}\}, \mathcal{C}_{2}=\mathcal{B}_{5,10} \cup\{\{1,2,3,4,9\},\{1,3,4,5,6\}\}, \\
\mathcal{C}_{3}=\mathcal{B}_{5,10} \cup\{\{1,3,4,5,6\},\{5,6,7,8,10\}\}, \mathcal{C}_{4}=\mathcal{B}_{5,10} \cup\{\{2,7,8,9,10\},\{5,6,7,8,10\}\} .
\end{gathered}
$$

All of them are of size 12 , and hence $\mathcal{A}_{I, \bar{I}}$ is a pure domain of rank $2 \cdot 5+\left(\begin{array}{l}2 \\ 2\end{array}\right)+\left(\begin{array}{l}2 \\ 2\end{array}\right)=12$, which agrees with the statement of Theorem 3.3 .

Next, we generalize Theorem 3.3 to the case of not necessarily complementary subsets. Namely, take any two subsets $I, J \in\left(\begin{array}{c}{[n]} \\ m\end{array}\right)$ and put $k:=|I \backslash J|=|J \backslash I|$. After we ignore the elements from $I \cap J$ and $\overline{J \cup I}$, we get two complementary sets $P, Q \in\left(\begin{array}{c}{[2 k]} \\ k\end{array}\right)$ (so $Q=\bar{P}$ ). We say that $I$ and $J$ form a balanced pair if the set $P$ is balanced.

Theorem 3.6 Let $I, J \in\left(\begin{array}{c}{[n]} \\ m\end{array}\right)$ form a balanced pair and let $P, Q, k$ be as above. Let $\left(p_{1}, p_{2}, \ldots, p_{2 r}\right)$ be the partition of the circle associated with $P$. Then $\mathcal{A}_{I, J}$ is a pure domain of rank

$$
m(n-m)-k^{2}+2 k+\sum_{i=1}^{2 r}\left(\begin{array}{c}
p_{i} \\
2
\end{array}\right) .
$$

Note that the additional term $m(n-m)-k^{2}$ is nothing but the difference of $\operatorname{ranks} \operatorname{rk}\left(\begin{array}{c}{[n]} \\ m\end{array}\right)-\operatorname{rk}\left(\begin{array}{c}{[2 k]} \\ k\end{array}\right)$. We conclude this section with the following theorem:

Theorem 3.7 Let $I, J, P, Q, k$ be as in Theorem 3.6 Then $d(I, J)=1+k^{2}-2 k-\sum_{i=1}^{2 r}\left(\begin{array}{c}p_{i} \\ 2\end{array}\right)$. If I and $J$ are not balanced then $d(I, J) \leq 1+k^{2}-2 k-\sum_{i=1}^{2 r}\left(\begin{array}{c}p_{i} \\ 2\end{array}\right)$.

The first part of this theorem follows from Theorem 3.6 For the second part, we have an even stronger upper bound which we omit here.

\section{Pure domains inside and outside a simple closed curve}

In this section we discuss the approaches of (Oh et al. 2015, Section 9) and Danilov et al. (2014) regarding domains inside and outside simple closed curves. We start by defining a map that appears in both of the papers and justifies the geometric intuition that we are using, for example, while thinking about simple closed curves. Let us fix $n$ vectors $\xi_{1}, \xi_{2}, \ldots, \xi_{n} \in \mathbb{R}^{2}$ at the vertices of a convex n-gon in clockwise order. Define:

$$
Z_{n}=\left\{\lambda_{1} \xi_{1}+\ldots+\lambda_{n} \xi_{n} \mid 0 \leq \lambda_{i} \leq 1, i=1,2, \ldots, n\right\} .
$$

We identify a subset $I \subset[n]$ with the point $\sum_{i \in I} \xi_{i}$ in $Z_{n}$. Note that if two subsets $I$ and $J$ are weakly separated, then the corresponding points are different. Now if we have a sequence $\mathcal{S}=\left(S_{0}, S_{1}, \ldots, S_{r}=\right.$ $S_{0}$ ) of subsets, we can always view it as a piecewise linear closed curve $\zeta_{\mathcal{S}}$ obtained by concatenating the line-segments connecting consecutive points $S_{i-1}$ and $S_{i}$ for $i=1,2 \ldots, r$. An important instance of such curves are generalized cyclic patterns. (iii)

Definition 4.1 Danilov et al. (2014) A generalized cyclic pattern is a sequence $\mathcal{S}=\left(S_{1}, S_{2}, \ldots, S_{r}=\right.$ $\left.S_{0}\right)$ of pairwise distinct and weakly separated subsets of $[n]$ such that

\footnotetext{
(iii) We will need them only for the case when all sets from $\mathcal{S}$ have the same size, so we give simplified versions of the definitions and a theorem from Danilov et al. (2014).
} 
1. The sets in $\mathcal{S}$ all have the same size;

2. $\left|S_{i-1} \triangle S_{i}\right|=2$ for $i=1, \ldots, r$.

For a generalized cyclic pattern $\mathcal{S} \subset\left(\begin{array}{c}{[n]} \\ k\end{array}\right)$, let $\mathcal{D}_{\mathcal{S}}:=\left\{X \in\left(\begin{array}{c}{[n]} \\ k\end{array}\right) \mid X\right.$ is weakly separated from $\left.\mathcal{S}\right\}$. All the generalized cyclic patterns discussed in this work correspond to non self-intersecting curves. For more details regarding criteria for non-self intersection, see Danilov et al. (2014). The following two theorems summarize important properties of generalized cyclic patterns. Let $\mathcal{S}$ be a generalized cyclic pattern for which the curve $\zeta_{\mathcal{S}}$ is non-self intersecting. Then $\zeta_{\mathcal{S}}$ subdivides $Z_{n}$ into two closed regions $\mathcal{R}_{\mathcal{S}}^{\text {in }}$ and $\mathcal{R}_{\mathcal{S}}^{\text {out }}$ such that $\mathcal{R}_{\mathcal{S}}^{\text {in }} \cap \mathcal{R}_{\mathcal{S}}^{\text {out }}=\zeta_{\mathcal{S}}$ and $\mathcal{R}_{\mathcal{S}}^{\text {in }} \cup \mathcal{R}_{\mathcal{S}}^{\text {out }}=Z_{n}$. Let us define

$$
\mathcal{D}_{\mathcal{S}}^{\text {in }}=\mathcal{D}_{\mathcal{S}} \cap \mathcal{R}_{\mathcal{S}}^{\text {in }} \text { and } \mathcal{D}_{\mathcal{S}}^{\text {out }}=\mathcal{D}_{\mathcal{S}} \cap \mathcal{R}_{\mathcal{S}}^{\text {out }}
$$

Theorem 4.2 (Danilov et al. (2014)) Let $\mathcal{S}$ be a generalized cyclic pattern for which the curve $\zeta_{\mathcal{S}}$ is nonself intersecting. Then the domains $\mathcal{D}_{\mathcal{S}}^{\text {in }}$ and $\mathcal{D}_{\mathcal{S}}^{\text {out }}$ are pure. Moreover, for every $X \in \mathcal{D}_{\mathcal{S}}^{\text {in }}$ and $Y \in \mathcal{D}_{\mathcal{S}}^{\text {out }}$, $X$ and $Y$ are weakly separated.

An important instance of generalized cyclic patterns, called Grassmann necklaces, was studied in $\mathrm{Oh}$ et al. (2015) and Postnikov (2006). For the case of Grassmann necklaces, the ranks of $\mathcal{D}_{\mathcal{S}}^{\text {in }}$ and $\mathcal{D}_{\mathcal{S}}^{\text {out }}$ can be calculated explicitly. We discuss here only connected necklaces and some notions associated with them. For the general case, we refer the reader to Oh et al. (2015).

Definition 4.3 A generalized cyclic pattern $\mathcal{S}=\left(S_{1}, S_{2}, \ldots, S_{r}=S_{0}\right)$ forms a connected Grassmann necklace iffor all $i \in[n], S_{i+1}=S_{i} \backslash\{i\} \cup\{j\}$ for some $j \in[n]$. $\mathcal{S}$ can be recorded through a decorated permutation $\pi:[n] \rightarrow[n]$ for which $\pi(i)=j$ if $S_{i+1}=S_{i} \backslash\{i\} \cup\{j\}$.

For $i, j \in[n],\{i, j\}$ forms an alignment in $\pi$ if $(i, \pi(i), \pi(j), j)$ are cyclically ordered (and all distinct). The number of alignments in $\pi$ is denoted by $\mathrm{al}(\pi)$, and the length $\ell(\pi)$ is defined to be $k(n-k)-\mathrm{al}(\pi)$.

This definition applies only to connected necklaces and describes how to associate a decorated permutation with a given Grassmann necklace. We refer the reader to Oh et al. (2015) for the general case, as well as construction of the inverse of this map. We use the notation $\mathcal{I}$ for Grassmann necklaces and define $\ell(\mathcal{I})$ to be $\ell(\pi)$, where $\pi$ is the associated decorated permutation of $\mathcal{I}$.

Theorem 4.4 Oh et al. (2015) Let us fix a Grassmann necklace $\mathcal{I}$. Then $\mathcal{D}_{\mathcal{I}}^{\text {in }}$ is a pure domain of rank $\ell(\mathcal{I})+1$. Any two maximal weakly separated collections in $\mathcal{D}_{\mathcal{I}}^{\text {in }}$ are linked by a sequence of mutations.

Note that Theorem 2.4 is a special case of Theorem 4.4 by setting $\mathcal{I}:=\mathcal{B}_{k, n}$. The decorated permutation that is associated with such $\mathcal{I}$ is $\tau_{k, n}$, which sends $i \in[n]$ to $i+k \in[n]$. We view permutations as maps $[n] \rightarrow[n]$, so if $\sigma$ and $\pi$ are two permutations of $[n]$, then $(\sigma \circ \pi)(i)=\sigma(\pi(i))$. Finally, we write each permutation in one-line notation as follows: $\sigma=(\sigma(1), \sigma(2), \ldots, \sigma(n))$.

Definition 4.5 Let $I \in\left(\begin{array}{c}{[2 k]} \\ k\end{array}\right)$ and let $\left(p_{1}, \ldots, p_{2 r}\right)$ be the partition of the circle associated with I. Define

$$
\tau_{I}:=\left(p_{1}, p_{1}-1, \ldots, 1, p_{1}+p_{2}, p_{1}+p_{2}-1, \ldots, p_{1}+1, \ldots, 2 k, 2 k-1, \ldots, 2 k-p_{2 r}+1\right) .
$$

We say that the permutation $\tau_{I} \circ \tau_{k, 2 k}$ is the canonical decorated permutation associated with $\mathcal{A}_{I, \bar{I}}$. We denote by $\mathcal{I}\left(\tau_{I} \circ \tau_{k, 2 k}\right)$ the Grassmann necklace that corresponds to $\tau_{I} \circ \tau_{k, 2 k}$ and call it the canonical Grassmann necklace associated with $I$. 
Example 4.6 Let $I=\{1,2,4,6,8\}$ be as in Examples 3.2 and 3.5. Then $\tau_{I}=(2,1,3,4,5,6,7,8,10,9)$,

$$
\tau_{5,10}=(6,7,8,9,10,1,2,3,4,5) \text { and } \tau_{I} \circ \tau_{5,10}=(6,7,8,10,9,2,1,3,4,5) .
$$

Therefore, the canonical Grassmann necklace associated with I is:

$$
\begin{array}{r}
\mathcal{I}\left(\tau_{I} \circ \tau_{5,10}\right)=\{\{1,2,3,4,5\},\{2,3,4,5,6\},\{3,4,5,6,7\},\{4,5,6,7,8\},\{5,6,7,8,10\}, \\
\{6,7,8,9,10\},\{2,7,8,9,10\},\{1,2,8,9,10\},\{1,2,3,9,10\},\{1,2,3,4,10\}\} .
\end{array}
$$

Remark 4.7 Note that $\{i, j\}$ is an alignment in $\tau_{I} \circ \tau_{k, 2 k}$ iff both $\tau_{k, 2 k}(i)$ and $\tau_{k, 2 k}(j)$ belong to the same interval $P_{m}$ for some $1 \leq m \leq 2 r$. Therefore,

$$
\ell\left(\mathcal{I}\left(\tau_{I} \circ \tau_{k, 2 k}\right)\right)=k^{2}-\sum_{i=1}^{2 r}\left(\begin{array}{c}
p_{i} \\
2
\end{array}\right) .
$$

Next, we introduce the notion of an isomorphism between two generalized cyclic patterns.

Definition 4.8 Two generalized cyclic patterns $\mathcal{S}=\left(S_{0}, S_{1}, \ldots, S_{r}=S_{0}\right)$ and $\mathcal{C}=\left(C_{0}, C_{1}, \ldots, C_{r}=C_{0}\right)$ with $S_{i}, C_{i} \subset[n]$ are called isomorphic if there exists a permutation $\gamma:[n] \rightarrow[n]$ such that:

- $\gamma\left(C_{i}\right)=S_{i}$ for $i=0 \ldots r$;

- $\gamma$ is a bijection $\mathcal{D}_{\mathcal{C}}^{\text {in }} \rightarrow \mathcal{D}_{\mathcal{S}}^{\text {in }}$;

- Two subsets $A, B \in \mathcal{D}_{\mathcal{C}}^{\text {in }}$ are weakly separated iff $\gamma(A)$ and $\gamma(B)$ are weakly separated.

Such a permutation $\gamma$ is called an isomoprhism between $\mathcal{S}$ and $\mathcal{C}$.

In other words, isomorphic generalized cyclic patterns differ by just a relabeling of elements of $[n]$. Note that the last two properties may not hold for $\mathcal{D}_{\mathcal{C}}^{\text {out }}$ and $\mathcal{D}_{\mathcal{S}}^{\text {out }}$. Clearly, if $\mathcal{S}$ and $\mathcal{C}$ are isomorphic, then the ranks of $\mathcal{D}_{\mathcal{S}}^{\text {in }}$ and $\mathcal{D}_{\mathcal{C}}^{\text {in }}$ are equal.

\section{Sketch of the proof}

In this section we sketch the proof of Theorem 3.3 The proof idea is to show that if $I$ is balanced and $\mathcal{W} \subset \mathcal{A}_{I, \bar{I}}$ is a maximal weakly separated collection, then $\mathcal{W} \subset \mathcal{D}_{\mathcal{S}_{\mathcal{W}}}^{\text {out }}$ for some generalized cyclic pattern $\mathcal{S}_{\mathcal{W}} \subset \mathcal{W}$. While $\mathcal{S}_{\mathcal{W}}$ depends on $\mathcal{W}$, all generalized cyclic patterns of this form will be shown to be isomorphic. In particular, all of them are isomorphic to the canonical Grassmann necklace associated with $I$. As a conclusion, we find that $\operatorname{rk} \mathcal{D}_{\mathcal{S}_{\mathcal{W}}}^{\text {out }}$ is independent of $\mathcal{W}$, and its value equals the one given in (3). This will imply that $\mathcal{A}_{I, \bar{I}}$ is a pure domain.

Example 5.1 Let I be as in example 3.5. There are four maximal weakly separated collections in $\mathcal{A}_{I, \bar{I}}$ given by $\mathcal{C}_{1}, \mathcal{C}_{2}, \mathcal{C}_{3}, \mathcal{C}_{4}$. Figure 2 depicts their embedding in the plain, as described in the previous section. Each one of these collections lies outside of a curve formed by a generalized cyclic pattern. The generalized cyclic pattern that corresponds to $\mathcal{C}_{4}$ is exactly $\mathcal{I}\left(\tau_{I} \circ \tau_{5,10}\right)$, the canonical Grassmann necklace associated with I (see Example 4.6 for the description of this necklace). The generalized cyclic patterns corresponding to $\mathcal{C}_{1}, \mathcal{C}_{2}$ and $\mathcal{C}_{3}$ are isomorphic to $\mathcal{I}\left(\tau_{I} \circ \tau_{5,10}\right)$. 

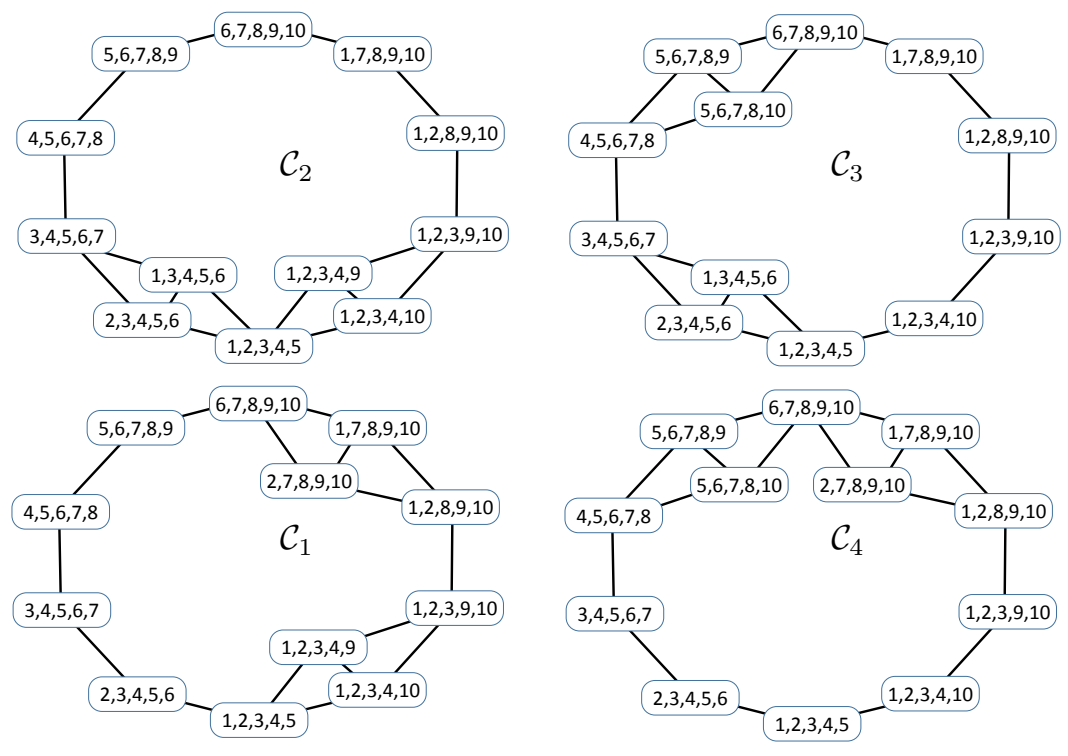

Fig. 2: The embedding of $\mathcal{C}_{1}, \mathcal{C}_{2}, \mathcal{C}_{3}, \mathcal{C}_{4}$.

One of the main ingredients of the proof is the following "left-right purity phenomenon" which is interesting in its own right.

Definition 5.2 For a positive integer $n$, denote by $\mathcal{L} \mathcal{R}([0, n])$ the collection of all subsets $I \subset[0, n]:=$ $\{0,1, \ldots, n\}$ such that I contains exactly one of the elements $0, n$.

Then we obtain the following description of maximal weakly separated collections inside $\mathcal{L} \mathcal{R}([0, n])$ :

Theorem 5.3 1. The domain $\mathcal{L} \mathcal{R}([0, n])$ is a pure domain of rank $\left(\begin{array}{c}n \\ 2\end{array}\right)+n+1=\operatorname{rk} 2^{[n]}$;

2. For each maximal weakly separated collection $M \subset \mathcal{L} \mathcal{R}([0, n])$ and for all $m=0, \ldots, n-1$ there is a unique set $S_{m} \subset[n-1]$ of size $m$ such that both $S_{m} \cup\{0\}$ and $S_{m} \cup\{n\}$ belong to M. For these sets, we have $\emptyset=S_{0} \subset S_{1} \subset \cdots \subset S_{n-1}=[n-1]$.

Remark 5.4 The second part of Theorem 5.3 follows directly from (Leclerc and Zelevinsky, 1998, Corollary 3.4). An almost identical but slightly different notion appears in (Danilov et al. 2010. Definition 6) as a left-right pair. However none of these papers mentions the purity of the corresponding domain.

Example 5.5 Let $\mathcal{C}=\{\{0\},\{0,1\},\{0,1,2\},\{0,1,2,3\},\{4\},\{3,4\},\{2,3,4\},\{1,2,3,4\},\{0,2,3\},\{0,2\},\{2,4\}\}$ be a maximal weakly separated collection in $\mathcal{L R}([0,4])$. We have $|\mathcal{C}|=11=\left(\begin{array}{l}4 \\ 2\end{array}\right)+4+1$ and the sequence $\left(S_{0} \subset S_{1} \subset S_{2} \subset S_{3}\right)$ provided by Theorem 5.3 is $(\emptyset \subset\{2\} \subset\{2,3\} \subset\{1,2,3\})$.

The next lemma describes the structure of the elements in $\mathcal{A}_{I, \bar{I}}$.

Lemma 5.6 Let $I \subset\left(\begin{array}{c}{[2 k]} \\ k\end{array}\right)$ be balanced, and let $\left\{P_{i}\right\}_{i=1}^{2 r}$ be the intervals associated with I. Then $U \in$ $\mathcal{A}_{I, \bar{I}}$ iff $U$ satisfies

$$
\left(P_{i+1} \cup P_{i+2} \cup \cdots \cup P_{j-1}\right) \subset U \subset P_{i} \cup\left(P_{i+1} \cup P_{i+2} \cup \cdots \cup P_{j-1}\right) \cup P_{j}
$$


for some $i$ and $j$.

If such $i$ and $j$ exist then they can be (uniquely) chosen so that $P_{i} \cap U$ and $P_{j} \cap U$ are nonempty. In this case $P_{i}$ and $P_{j}$ are called (resp., left and right) endpoints of $U$. For example, if $I$ is as in Example 3.2 then $\{1,3,4,5,6\} \in \mathcal{A}_{I, \bar{I}}$. Its endpoints are $\{1,2\}$ and $\{6\}$.

The following lemma is an application of the Left-Right Purity (Theorem 5.3. It explains how to construct the generalized cyclic pattern $S_{\mathcal{W}}$ mentioned in the beginning of the section.

Lemma 5.7 Let I be as in Lemma 5.6. and let $\mathcal{W} \subset \mathcal{A}_{I, I}$ be maximal weakly separated collection. Then for each $1 \leq i \leq 2 r$, there exists a sequence of subsets $\emptyset=S_{0}^{i} \subset S_{1}^{i} \subset \cdots \subset S_{p_{i}}^{i}=P_{i}$ satisfying the following properties:

(a) for any $1 \leq i \leq 2 r, 0 \leq t \leq p_{i}$ and for any $U \in \mathcal{W}$ the sets $U \cap P_{i}$ and $S_{t}^{i}$ are weakly separated;

(b) any set in $\mathcal{W}$ is weakly separated from any $S \in \mathcal{A}_{I, \bar{I}}$ such that if $P_{i}$ and $P_{j}$ are the endpoints of $S$ then $S \cap P_{i}=S_{t}^{i}$ and $S \cap P_{j}=S_{u}^{j}$ for some $t \leq p_{i}$ and $u \leq p_{j}$.

Now consider all the sets $U \in \mathcal{A}_{I, \bar{I}}$ satisfying property (b) Note that they form a generalized cyclic pattern: if $S_{m}=S_{t}^{i} \cup P_{i+1} \cup \cdots \cup P_{j-1} \cup S_{u}^{j}$ is such a set (with $S_{t}^{i}$ and $S_{u}^{j}$ nonempty) then it is followed by the set

$$
S_{m+1}= \begin{cases}S_{t-1}^{i} \cup P_{i+1} \cup \cdots \cup P_{j-1} \cup S_{u+1}^{j}, & \text { if } u<p_{j} \\ S_{t-1}^{i} \cup P_{i+1} \cup \cdots \cup P_{j-1} \cup P_{j} \cup S_{1}^{j+1}, & \text { if } u=p_{j} .\end{cases}
$$

Every subset $S \in \mathcal{A}_{I, \bar{I}}$ satisfying property[(b) appears somewhere in this sequence $\mathcal{S}_{\mathcal{W}}:=\left(S_{1}, S_{2}, \ldots, S_{2 k}\right)$. Here $S_{1}$ is chosen so that its left endpoint is $P_{1}$ and $S_{1} \cap P_{1}=P_{1}$. From (Danilov et al., 2014, Theorem 7.4), we know that the domains $\mathcal{D}_{\mathcal{S}_{\mathcal{W}}}^{\text {in }}$ and $\mathcal{D}_{\mathcal{S}_{\mathcal{W}}}^{\text {out }}$ form a complementary pair and thus

$$
\operatorname{rk} \mathcal{D}_{\mathcal{S}_{\mathcal{W}}}^{\text {in }}+\operatorname{rk} \mathcal{D}_{\mathcal{S}_{\mathcal{W}}}^{\text {out }}=1+k^{2}+|\mathcal{S}|=1+k^{2}+2 k .
$$

To calculate the ranks of $\mathcal{D}_{\mathcal{S}}^{\text {in }}$ and $\mathcal{D}_{\mathcal{S}}^{\text {out }}$, we prove the following theorem:

Theorem 5.8 The canonical Grassmann necklace associated with I is isomorphic to $\mathcal{S}_{\mathcal{W}}$.

By Theorem 5.8 and Remark 4.7 it follows that $\operatorname{rk} \mathcal{D}_{\mathcal{S}_{\mathcal{W}}}^{\text {in }}=1+\ell\left(\tau_{A} \circ \tau_{k, 2 k}\right)=1+k^{2}-\sum_{i=1}^{2 r}\left(\begin{array}{c}p_{i} \\ 2\end{array}\right)$. Substituting this into 5 yields $\mathrm{rk} \mathcal{D}_{\mathcal{S}_{\mathcal{W}}}^{\text {out }}=2 k+\sum_{i=1}^{2 r}\left(\begin{array}{c}p_{i} \\ 2\end{array}\right)$.

Finally, using the geometric definition of domains $\mathcal{D}_{\mathcal{S}_{\mathcal{W}}}^{\text {in }}$ and $\mathcal{D}_{\mathcal{S}_{\mathcal{W}}}^{\text {out }}$ given in Section 4 we show:

Lemma 5.9

$$
\mathcal{W} \subset \mathcal{D}_{\mathcal{S}_{\mathcal{W}}}^{\text {out }} \subset \mathcal{A}_{I, \bar{I}}
$$

This lemma implies that $\mathcal{W}$ is a maximal weakly separated collection inside $\mathcal{D}_{\mathcal{S}}^{\text {out }}$ and thus $|\mathcal{W}|=\operatorname{rk} \mathcal{D}_{\mathcal{S}}^{\text {out }}$, which completes the proof of Theorem 3.3

\section{Mutation distance and sets with four associated intervals}

In the previous sections, we found the exact value of $d(\cdot, \cdot)$ for balanced pairs, and an upper bound for unbalanced pairs. In this section, we evaluate the exact value of $d(\cdot, \cdot)$ for a wide family of unbalanced pairs. Recall that for an even positive integer $t, \mathcal{P}_{t}$ is the collection of all sets $I$ for which $I$ and $\bar{I}$ partition the circle into $t$ intervals. In this section we concentrate on the structure of $\mathcal{P}_{4}$. This case is particularly 
interesting, since $t=4$ is the minimal $t$ for which $I, \bar{I} \in \mathcal{P}_{t}$ are not weakly separated. While sets $I \in \mathcal{P}_{4}$ can never be balanced, we were still able to find the exact value of $d(I, \bar{I})$ as well as the maximal possible cardinality of a weakly separated collection in $\mathcal{A}_{I, \bar{I}}$. Moreover, we also find the value of $D(I, \bar{I})$, the mutation distance between $I$ and $\bar{I}$ introduced in Farber and Postnikov (2015). The following definition and problem were introduced in Farber and Postnikov (2015).

Definition 6.1 Let $I, J \in\left(\begin{array}{c}{[n]} \\ k\end{array}\right)$ be any two k-element subsets in $[n]$. Define the mutation distance $D(I, J)$ to be the minimal number of mutations needed to transform a maximal weakly separated collection $\mathcal{C}_{1} \subset$ $\left(\begin{array}{c}{[n]} \\ k\end{array}\right)$ that contains I into a maximal weakly separated collection $\mathcal{C}_{2} \in\left(\begin{array}{c}{[n]} \\ k\end{array}\right)$ that contains $J$.

Problem 6.2 How to calculate the mutation distance between any I and J, and how to find the shortest chain of mutations between maximal weakly separated collections containing these subsets?

Clearly, $D(I, J)=0$ iff $I$ and $J$ are weakly separated. In addition, $d(I, J) \leq D(I, J)$ for any pair $I, J \in\left(\begin{array}{c}{[n]} \\ k\end{array}\right)$. Combining this observation with Theorem 3.7 leads to the following corollary:

Corollary 6.3 Let $I, J, P, Q, k$ be as in Theorem 3.6 Then $D(I, J) \geq 1+k^{2}-2 k-\sum_{i=1}^{2 r}\left(\begin{array}{c}p_{i} \\ 2\end{array}\right)$.

We proceed to the results about $\mathcal{P}_{4}$.

Theorem 6.4 Let $I \in\left(\begin{array}{c}{[2 k]} \\ k\end{array}\right)$ for which $\left(p_{1}, p_{2}, p_{3}, p_{4}\right)$ is the partition of the circle associated with I. Then a maximal weakly separated collection in $\mathcal{A}_{I, \bar{I}}$ is of size at most $2 k+\sum_{i=1}^{4}\left(\begin{array}{c}p_{i} \\ 2\end{array}\right)$, and this bound it tight.

Corollary 6.5 Let I be as in Theorem 6.4 Then $d(I, \bar{I})=1+k^{2}-2 k-\sum_{i=1}^{2 r}\left(\begin{array}{c}p_{i} \\ 2\end{array}\right)$.

Theorem 6.6 Let I be as in Theorem 6.4 and assume without loss of generality that $p_{1} \geq \max \left(p_{2}, p_{3}, p_{4}\right)$ (we can always achieve that by rotation and switching the roles of $I$ and $\bar{I}$ ). Then $D(I, \bar{I})=p_{2} p_{3} p_{4}-2\left(\begin{array}{c}p_{3}+1 \\ 3\end{array}\right)$.

Remark 6.7 For $I \in \mathcal{P}_{4}$, Theorem 6.6 implies that $D(I, \bar{I})$ is a (piecewise) polynomial of degree 3, while Corollary 6.5 implies that $d(I, I)$ is a polynomial of degree 2. It would be interesting to figure out whether both $d(\cdot, \cdot)$ and $D(\cdot, \cdot)$ are piecewise polynomials (in the parameters $\left\{p_{i}\right\}_{i=1}^{2 r}$ ) for the general case. In addition, Theorems 6.4 and 3.3 give identical values for the maximal cardinality of a maximal weakly separated collection in $\mathcal{A}_{I, \bar{I}}$. However, for $I \in \mathcal{P}_{4}, \mathcal{A}_{I, \bar{I}}$ is not necessarily pure, so there might be a maximal (by inclusion) weakly separated collection in $\mathcal{A}_{I, \bar{I}}$ with smaller cardinality.

The main tool that is used in the proof of these results is a certain projection of weakly separated collections in $\mathcal{A}_{I, \bar{I}}$ onto $\mathbb{R}^{3}$, and the relation of this projection with mutation sequences. This projection was introduced in Farber and Postnikov (2015), where using computer experiments, the values of $D(\cdot, \cdot)$ were found for small cases. Here we fully explore the properties of this projection, and this enables us to find $D(I, \bar{I})$ and $d(I, \bar{I})$ for any $I \in \mathcal{P}_{4}$.

Definition 6.8 Given a partition $\left(P_{1}, P_{2}, P_{3}, P_{4}\right)$ of a circle into four intervals, we define the projection $\phi_{P_{1}, P_{2}, P_{3}, P_{4}}: 2^{[n]} \rightarrow \mathbb{R}^{4}$ such that for $S \subset 2^{[n]}, \phi_{P_{1}, P_{2}, P_{3}, P_{4}}(S)=\left(\left|S \cap P_{1}\right|,\left|S \cap P_{2}\right|,\left|S \cap P_{3}\right|,\left|S \cap P_{4}\right|\right)$.

For a weakly separated collection $\mathcal{C} \subset\left(\begin{array}{c}{[n]} \\ k\end{array}\right), \phi_{P_{1}, P_{2}, P_{3}, P_{4}}(\mathcal{C})$ is a certain set of lattice points that all belong to the three-dimensional hyperplane $x_{1}+x_{2}+x_{3}+x_{4}=k$. Let

$$
\alpha_{1}=(0,0,-1,1), \alpha_{2}=(0,1,-1,0), \alpha_{3}=(-1,1,0,0), \alpha_{4}=(-1,0,0,1) \text {. }
$$


In order to prove the results, we take $P_{1}, P_{2}, P_{3}$ and $P_{4}$ to be the intervals associated with $I$. Then for any pair of maximal weakly separated collections $\mathcal{C}_{1}, \mathcal{C}_{2} \subset\left(\begin{array}{c}{[2 k]} \\ k\end{array}\right)$ such that $I \in \mathcal{C}_{1}, \bar{I} \in \mathcal{C}_{2}$, we show that all the points in the projections $\phi_{P_{1}, P_{2}, P_{3}, P_{4}}\left(\mathcal{C}_{1}\right)$ and $\phi_{P_{1}, P_{2}, P_{3}, P_{4}}\left(\mathcal{C}_{2}\right)$ must lie outside, or on the surface, of the square pyramids $Q_{1}=\left\{\phi(I)+\sum_{i=1}^{4} t_{i} \alpha_{i} \mid t_{i} \geq 0 \forall i\right\}$ and $Q_{2}=\left\{\phi(\bar{I})-\sum_{i=1}^{4} t_{i} \alpha_{i} \mid t_{i} \geq 0 \forall i\right\}$ respectively. We proceed by showing that any mutation either leaves the projection unchanged, or applies an "octahedron move", that is, replacing upper half of the octahedron by its lower half. This enables us to show that $d(I, \bar{I})$ equals to the number of the lattice points on the part of the surface of $Q_{1}$ that lies inside $Q_{2}$, while $D(I, \bar{I})$ equals to the number of integer lattice points inside the intersection of $Q_{1}$ and $Q_{2}$. These quantities are exactly the values given in Corollary 6.5 and Theorem 6.6 Figure 3 depicts a a shortest mutation sequence between two pyramids.
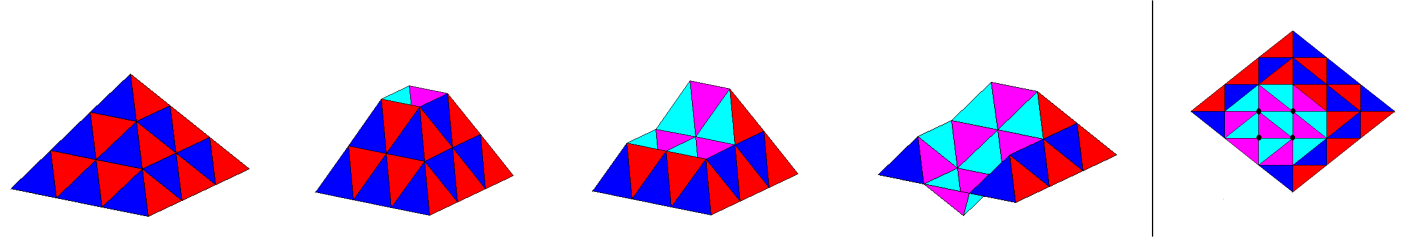

Fig. 3: An example of shortest sequence of mutations on the left and the surface of $Q_{1}$ that lies inside $Q_{2}$ on the right.

\section{References}

V. I. Danilov, A. V. Karzanov, and G. A. Koshevoy. On maximal weakly separated set-systems. $J$. Algebraic Combin., 32(4):497-531, 2010.

V. I. Danilov, A. V. Karzanov, and G. A. Koshevoy. Combined tilings and the purity phenomenon on separated set-systems. arXiv preprint arXiv:1401.6418, 2014.

M. Farber and A. Postnikov. Arrangements of equal minors in the positive Grassmannian. To appear in Advances in Mathematics (Zelevinsky issue), 2015. URL http://arxiv.org/pdf/1502. $01434 \mathrm{v} 1 \cdot \mathrm{pdf}$.

B. Leclerc and A. Zelevinsky. Quasicommuting families of quantum Plücker coordinates. In Kirillov's seminar on representation theory, volume 181 of Amer. Math. Soc. Transl. Ser. 2, pages 85-108. Amer. Math. Soc., Providence, RI, 1998.

S. Oh and D. E. Speyer. Links in the complex of weakly separated collections. arXiv preprint arXiv:1405.5191, 2014.

S. Oh, A. Postnikov, and D. E. Speyer. Weak separation and plabic graphs. Proc. Lond. Math. Soc. (3), 110(3):721-754, 2015.

A. Postnikov. Total positivity, Grassmannians, and networks. arXiv preprint math/0609764, 2006.

J. Scott. Quasi-commuting families of quantum minors. J. Algebra, 290(1):204-220, 2005. 